\title{
Stability Analysis of Slope Based on Finite Element Method
}

\author{
Bo He, Hongcai Zhang
}

\begin{abstract}
Mechanical \& Electrical Engineering College Shenyang Aerospace University Shenyang, Liaoning Province, China

Department of mechanical engineering Armor Technique Institute of PLA Changchun, Jilin Province, China
\end{abstract}

\begin{abstract}
Based on the finite element software as calculation tool, Mohr-Coulomb yield criterion and Drucker-Prager yield criterion were converted to equivalent area circle yield criterion, the elastic-plastic large deformation finite element method (FEM) was adopted, at the same time strength reduction technology combined with convergence criterion, catastrophe criterion and plastic zone penetrability criterion was used, and the problem of 2-D slope stability was analysed. Then based on finite element reduction strength method and three criterions, the homogeneous slope safety factors were calculated. The results showed that equivalent area circle D - P yield criterion was suitable for slope stability analysis and that strength reduction technology could be applied in simple homogeneous side slope stability analysis.
\end{abstract}

Index Terms: Man-made object detection; image interpretation; feature extraction; clustering

(0) 2012 Published by MECS Publisher. Selection and/or peer review under responsibility of the Research Association of Modern Education and Computer Science.

\section{Introduction}

Slopes include the natural and the artificial slopes [1]. The disasters of landslide and collapse often occur in China, and according to incomplete statistics, one serious disaster of landslide or collapse occurred every year on average in recent ten years [2]. Therefore, it is of great significance to study the problem of slope deformation and failure. Because the slope stability problem has already become the question which is often encountered in engineering, no matter what kind of geotechnical engineering, ensuring slope stability and preventing slope instability is the key, thus calculation methods of the slope stability analysis has got great development in this condition. The popular existing slope stability analysis methods are mainly the slice method based on the limit equilibrium theory and the numerical method based on the elastic-plastic theory [3,4]. The limit equilibrium method is a more traditional and mature method for the slope stability analysis and mainly includes the Fellenius method, the Bishop method, the Janhu method, the Morgenstern-Price method, the Spencer method, the slide wedge method, the imbalance thrust force method, the Sarma method [5,6].

With further developing of the computer technology and calculating methods, the finite element method, the boundary element method and the discrete element method and the finite difference method are widely adopted in the slope stability calculation, among which the finite element method is used the most widely. In this paper, Mohr-Coulomb yield criterion and Drucker-Prager yield criterion will be converted to equivalent area circle yield criterion, the elastic-plastic large deformation finite element method (FEM) will be adopted, at the same 
time strength reduction technology combined with convergence criterion, catastrophe criterion and plastic zone penetrability criterion will be used, and the problem of 2-D slope stability will be analyzed.

\section{Calculation method}

\section{A. Yield Criterion of Soil}

In engineering the most commonly used is Mohr-Coulomb yield criterion (hereinafter referred to as M-C):

$\frac{1}{3} J_{1} \sin \varphi+\left[\cos \theta-\frac{1}{\sqrt{3}} \sin \theta \sin \varphi\right] \sqrt{J_{2}}=C \cos \varphi$

where, J1: the first invariant of stress tensor, J2: the second invariant of stress deviator tensor; $\theta$ : stress Lode angle.

The yield surface of M-C is an irregular six pyramid surface with singular vertices, and the derivative calculation is difficult or even not convergent. Therefore, scholars put forward the correction model in succession, such as Drucker-Prager yield surface (D-P criterion).

$$
f=\alpha J_{1}+\sqrt{J_{2}}-k=0 \text { or } \alpha J_{1}+\sqrt{J_{2}}=k .
$$

The equivalent area circle D-P yield criterion is used widely in the study of stability of circular slopes.

$$
\alpha=\frac{\sqrt{2 \sqrt{3}} \sin \varphi}{\sqrt{\pi\left(9-\sin ^{2} \varphi\right)}} ; k=\frac{6 \sqrt{\sqrt{3}} C \cos \varphi}{\sqrt{2 \pi\left(9-\sin ^{2} \varphi\right)}} .
$$

\section{B. Strength Reduction Theory}

The safety factor of the slope stability can be defined as soil shear strength reduced degree when the slope critical failure status is just reached, and it equals the ratio of the soil shear strength and the reduced soil shear strength of critical failure status.

The shear strength reduction factor is defined as the ratio of the maximum shear strength of slope soil and the actual shear stress of the slope under external loads, remaining the same external loads circumstances. The shear strength reduction coefficient is defined as the overall stability factor of slope safety, hence this safety coefficient can be considered to the strength reserve safety coefficient. Strength reduction concept can unify the strength reserve safety coefficient and the strength safety coefficient of the slope overall stability. And the finite element method may be used to calculated shear strength reduction factor without determination of the shape and position of failure surface.

In elastic-plastic finite element numerical analysis based on the concept of strength reduction, for a point in domain, according to the general definition of the Bishop safety coefficient and considering the shear strength, the Mohr-Coulomb failure criteria is expressed as:

$$
\tau_{f}=C+\sigma \tan \phi .
$$

where, $\mathrm{C}$ is the cohesive force of the soil; $\phi$ is the internal friction angle.

The safety factor of the appointed shear plane of this point is

$$
F=\frac{\tau_{f}}{\tau}=\frac{C+\sigma \tan \phi}{\tau}
$$

Assume that the shear failure of soil dose not occur, the actual shear stress in soils and the maximum shear strength are same, that is

$$
\tau=\tau_{f m}=\frac{\tau_{f}}{F}=\frac{C+\sigma \tan \phi}{F}=C_{m}+\sigma \tan \phi_{m} .
$$

So the actual shear stress in soils can be looked as the fold reduction shear strength indexes and the fold reduction shear strength indexes are respectively:

$$
C_{m}=\frac{C}{F} ; \phi_{m}=\arctan \frac{\tan \phi}{F} \text {. }
$$

First the fold reduction factor $\mathrm{F}$ was given a tentative value, then according to theory of strength reduction, the fold reduction shear strength indexes were obtained, and $\mathrm{F}$ was continuously changed until the computation was divergent. 


\section{Numerical Results and Discussion}

\section{A. Basic Model Parameters}

There was a homogeneous slope and the model was shown in Fig. 1 . Soil density $\rho=1827 \mathrm{~kg} / \mathrm{m} 3$, sticky gather strength $\mathrm{C}=10000 \mathrm{~Pa}$ and internal friction angle $\varphi=5^{\circ}$, angle of dilatancy was $0^{\circ}$, deformation modulus $\mathrm{E}=20 \mathrm{Mpa}$ and Poisson's ratio v=0.3, equivalent area D-P circle yield criterion was used, and the finite element model of the slope was shown in Fig.2.

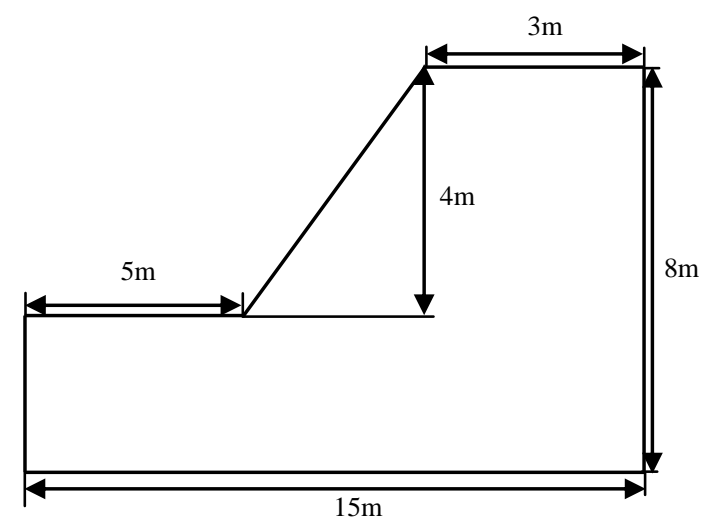

Fig.1 Calculation model

Fig.2 Finite element mode

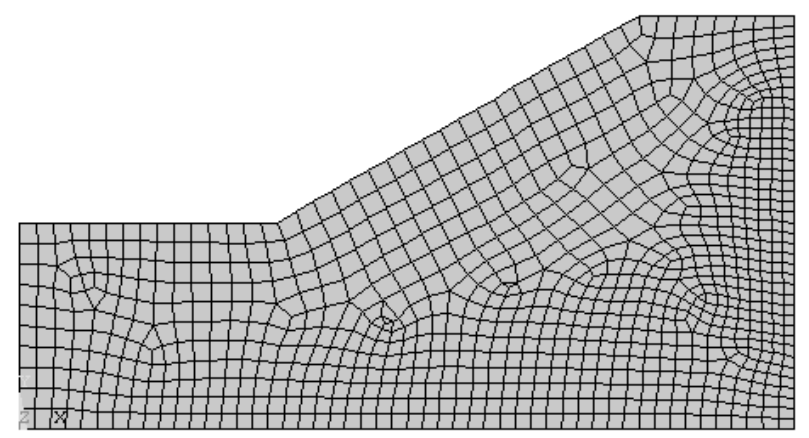

\section{B. Boundary Condition}

The right and the left sides of the slope were horizontally constrained, and the underside was completely fixed. For the natural slope, the external load was only the gravity.

\section{Calculation Results}

1) Criterion 1: Convergence criteria.

The iteration divergence of the finite element calculation of force or displacement is used as a marker of slope overall failure.

Based on the basic principle of finite element shear strength reduction, first 1.0 was taken as the initial fold reduction factor, the above material parameters were directly substituted into the finite element software, and the results showed that calculating was convergence and the slope was in a stable state. Then the fold reduction factor gradually increased by 0.1 and calculation was continuously carried out, when fold reduction factor reached 2.0 the calculating result was not convergence and the slope was in failure state. In order to make the calculating result more precise, between 1.9 and 2.0 the fold reduction factor gradually increased by 0.02 , and fold reduction calculation was continuously carried out, when fold reduction factor reached 1.98 the calculating result was convergence and the slope was in a stable state. But when fold reduction factor reached 1.985 the calculating result was not convergence and the slope was in failure state. Therefore, through the convergence criteria, the slope safety factor was 1.98.

2) Criterion 2: Catastrophe criteria. 
The infinite movements of the soil on the slope slide surface can be used to judge the failure of the slope, namely, slope will be in failure state when the variation of slope displacement appears saltation and unlimited development.

Similarly, based on the inscribed circle yield criterion, the relationship between the displacements of the most maximum, No. 92 node of slope top, No.100 node of slope bottom and No. 110 node of middle slope surface and the fold reduction factors was analysed. The result was shown in Table I.

TABLE IRELATIONSHIP BETWEEN DISPLACEMENTS AND REDUCTION FACTORS

\begin{tabular}{|c|c|c|c|c|}
\hline Reduction factors & \multicolumn{4}{|c|}{ Displacements (mm) } \\
\cline { 2 - 5 } & Maximum & No.100 node & No. 110 node & No. 92 node \\
\hline \multirow{3}{*}{1.0} & 30.5 & 6.7 & 13.9 & 26.1 \\
\cline { 2 - 5 } 1.1 & 33.5 & 7.0 & 14.2 & 28.1 \\
\cline { 2 - 5 } 1.2 & 36.8 & 7.5 & 14.6 & 29.9 \\
\cline { 2 - 5 } 1.3 & 40.4 & 8.2 & 15.0 & 31.9 \\
\cline { 2 - 5 } 1.4 & 45.2 & 9.4 & 15.8 & 34.7 \\
\cline { 2 - 5 } 1.5 & 52.4 & 12.6 & 17.5 & 46.0 \\
\cline { 2 - 5 } 1.6 & 65.9 & 22.1 & 21.9 & 62.4 \\
\cline { 2 - 5 } 1.8 & 102.2 & 49.4 & 141.3 & 290.3 \\
\cline { 2 - 5 } 1.9 & 322.4 & 219.3 & 251.9 & 310.8 \\
\cline { 2 - 5 } 1.92 & 510.7 & 372.3 & 277.9 & 332.5 \\
\cline { 2 - 5 } 1.94 & 540.4 & 425.5 & 297.2 & 355.2 \\
\cline { 2 - 5 } & 568.9 & 453.9 & & \\
\hline
\end{tabular}

From Table I, with the increasing of fold reduction factor, the maximum displacement and the displacements of No.100, 110 and 92 nodes changed slowly at first, when the fold reduction factor increased to1.7, the regularity of slow growth was still maintained, but when the fold reduction factor reached 1.9, the displacement of slope changed obviously and the slope was in instability state. According to the catastrophe criteria, the slope fold reduction factor was 1.92 .

3) Criterion 2: Plastic zone penetrability criteria.

The penetrability of generalized plastic strain or equivalent plastic strain from the slope top to the slope bottom is used as a marker of slope overall failure.

With the fold reduction factor changing continuously, the change of equivalent plastic strain was observed. And the results showed that plastic zone appeared firstly at slope bottom, then plastic region formed inside the slope, with the changing of the fold reduction factor, the plastic region was gradually expanded from the internal to surface. Due to the constraint of the surrounding rock mass, the slope was in stable state (shown in Fig.3). With continuous increase of the fold reduction factor, the plastic area of the slope obtained further development and formed a possible shear failure region, namely, it reached the critical damage status of slope. The status of $F=1.975$ was shown in Fig.4. The plastic strain within the slope was concentrated in destroying belt, but it remained stable. If the fold reduction factor continued to increase, the plastic area of the slope would be completely penetrated, causing the whole slope instability. So according to plastic zone penetrability criterion, the safety coefficient of slope was 1.975 .

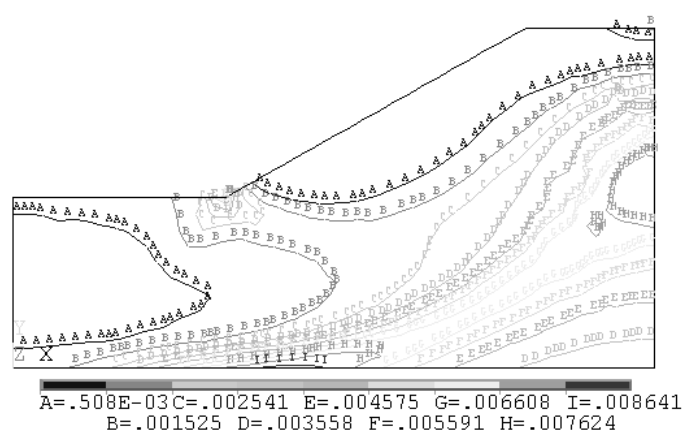

Fig. 3 Equivalent plastic stain isoline when $\mathrm{F}=1.5$ 


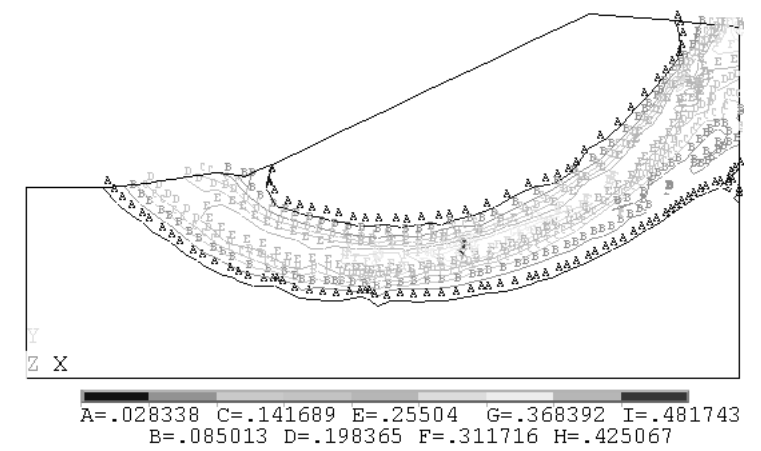

Fig.4 Equivalent plastic stain isoline when $\mathrm{F}=1.975$

\section{4. conclusion}

Through the above analysis and calculation the following conclusions could be made.

(1) The finite element method did not need to make any assumption and the calculating results were reliable. Finite element method could be used to analyze slope of complex shape and made of various materials without prior assumption of sliding surface.

(2) According to the slope stability theory based on the strength reduction and finite element analysis, fold reduction factor and the slope stability coefficient was the same, and through fold reduction of the strength of soil materials the stability of the slope was analyzed. The strength reduction coefficient would be the slope safety factor, when the convergence condition was not satisfied.

(3) According to three different instability criterions, we found that the corresponding safety factors obtained by the three different kinds of instability criterions were basically the same, that finite element shear strength reduction method could be used in engineering practice and that its application in simple homogeneous side slope stability analysis was feasible.

\section{References}

[1] H. Zheng, L. G. Tham, D. F. Liu, "On two definitions of the factor of safety commonly used in the finite element slope stability analysis." Computers and Geotechnics,Vol.33, no.3, pp.188-195, April 2006.

[2] Y.M. Cheng, T. Lansivaara, W.B. Wei, "Two-dimensional slope stability analysis by limit equilibrium and strength reduction methods." Computers and Geotechnics, Vol.34, no.3, pp.137-150, May 2007.

[3] K.S. Kahatadeniya, P. Nanakorn, K.M. Neaupane, "Determination of the critical failure surface for slope stability analysis using ant colony optimization." Engineering Geology, Vol.108, no. 1-2, pp.133-141, September 2009.

[4] Y.M. Cheng, H.T. Liu, W.B. Wei, "Location of critical three-dimensional non-spherical failure surface by NURBS functions and ellipsoid with applications to highway slopes." Computers and Geotechnics, Vol.32, no.6, pp.387-399, September 2005.

[5] W.B. Wei, Y.M. Cheng, L. Li, "Three-dimensional slope failure analysis by the strength reduction and limit equilibrium methods Original Research Article." Computers and Geotechnics, Vol. 1-2, no.6, Pages 70-80, January-March 2009.

[6] J. M. Duncan, "State of the art: Limit equilibrium and finite element analysis of slopes." Journal of Geotechnical Engineering, vol.122, no.7, pp.577-596, 1996. 